\title{
Wavefront Aberrations in Eyes With Acrysof Monofocal Intraocular Lenses
}

\author{
Prema Padmanabhan, MS; Geunyoung Yoon, PhD; Jason Porter, PhD; Srinivas K. Rao, FRCSEd; \\ Roy J, MSc; Mitalee Choudhury, BS
}

\section{ABSTRACT}

PURPOSE: To characterize and measure the ocular aberrations in eyes implanted with monofocal intraocular lenses (IOLs) and to study any correlation between postoperative aberrations and surgical factors.

METHODS: A Tscherning aberroscope was used to measure the wavefront aberrations of 62 eyes that had undergone phacoemulsification with the implantation of foldable monofocal Acrysof MA6OBM IOLs (Alcon Laboratories Inc, Ft Worth, Tex). The Zernike coefficients, measured with a pupil diameter of $6 \mathrm{~mm}$, were compared with those of a normal dataset of 82 eyes of healthy young myopes.

RESULTS: Spherical aberration $\left(Z_{4}^{0}\right)$ was the most predominant higher order aberration, with a mean value of $0.37 \pm 0.16 \mu \mathrm{m}$. A statistically significant linear relationship was noted between the magnitude of postoperative spherical aberration and the dioptric power of the IOL. The mean spherical aberration was 33 times more in the pseudophakic group than in normal young myopic eyes. The other major higher order aberrations were trefoil $\left(Z_{3}^{-3}\right)$ with a mean of $-0.13 \pm 0.22 \mu \mathrm{m}$ and vertical coma $\left(Z_{3}^{-1}\right)$ with a mean value of $-0.11 \pm 0.23$ $\mu \mathrm{m}$. On average, the root-mean-square of higher order aberrations in pseudophakic eyes was 2.1 times that in a normal population of young myopic eyes.

CONCLUSIONS: Eyes that undergo cataract surgery with monofocal IOL implantation suffer from significant higher order aberrations. The optical design of the IOL is most likely responsible for the increase in spherical aberration, the magnitude of which is a function of the dioptric power of the IOL. [J Refract Surg. 2006;22:237242.]

Journal of Refractive Surgery Volume 22 March 2006
$\mathrm{P}$

hacoemulsification, with the implantation of a foldable intraocular lens (IOL), has become the most popular technique for cataract surgery. ${ }^{1}$ The small self-sealing incision has reduced surgically induced astigmatism. ${ }^{2}$ The continuous curvilinear capsulorrhexis has increased the surgeon's ability to ensure a stable centration of the IOL. Precision in biometry and improvements in calculation of IOL power have enhanced the predictability of desired postoperative results. All of these optical and biomechanical factors are responsible for the qualification of cataract surgery as a "refractive procedure."

Until recently, the term "refractive procedure" has been used solely for spherocylindrical corrections. We now know that optical imperfections other than spherocylindrical refractive errors influence visual function..$^{3-5}$ The introduction of wavefront sensors into clinical practice has given the clinician a powerful tool to measure and examine these aberrations and thereby describe retinal image quality in more definitive terms. ${ }^{6}$

Most IOLs marketed today have better optical quality than that of a healthy crystalline lens. ${ }^{7-10}$ However, inserting IOLs of better optical quality does not directly translate to having optimum optical performance once inside the human eye. ${ }^{11-13}$ This discrepancy can be explained by the fact that, in the laboratory, the optical quality of the IOL is tested as a single unit, whereas the optical quality of the entire eye is mainly governed by both the cornea and the IOL. Artal et al ${ }^{14,15}$ have shown that aberrations in the young cornea can be partially compensated by lenticular aberrations, resulting in an eye that has better overall optical quality than either two components alone. For this reason, inserting an optically "perfect" IOL into

From Medical \& Vision Research Foundation, Tamil Nadu, India (Padmanabhan, Rao, J, Choudhury); and the Center for Visual Science, University of Rochester, Rochester, NY (Yoon, Porter).

The authors have no financial or proprietary interest in the materials presented herein.

Correspondence: Prema Padmanabhan, MS, Cornea Service, Sankara Nethralaya, 18 College $R d$, Chennai 600 006, Tamil Nadu, India. Tel: 9144 28271616; Fax: 9144 28254180; E-mail: drpp@snmail.org

Received: March 28, 2005

Accepted: April 25, 2005 
the eye will not yield a perfect overall system, devoid of aberrations. Rather, the ideal IOL should contain aberrations that are equal in magnitude and opposite in sign to those inherent in an individual's cornea.

Other possible causes for a suboptimal optical performance of an IOL implanted into an eye could be due to decentration of the IOL and possible changes in corneal shape induced by the cataract wound.

Clinical studies on the visual acuity and contrast sensitivity of patients with monofocal or multifocal IOLs have been performed. ${ }^{16,17}$ These, however, are measures of both the optical and neural processes of vision and do not directly describe the optical performance of the IOL. Artal et $\mathrm{al}^{18}$ used the double-pass measurements of the modulation transfer function (MTF) in eyes implanted with IOLs. Although the MTF is a good metric of retinal image quality, wavefront aberration may be a more complete description of optical quality as it contains phase information as well. ${ }^{12}$ The optical aberrations of eyes implanted with an IOL were only recently measured in vivo by Miller et al ${ }^{19}$ in 11 patients using the Shack-Hartmann sensor and by Barbero et $\mathrm{al}^{12}$ in 9 eyes using the laser ray tracing technique. It is hoped that the present study, with its relatively larger data set of 62 eyes, will add statistical value to the observations made. Although Miller et $\mathrm{al}^{19}$ and Barbero et $\mathrm{al}^{12}$ observed an increase in positive spherical aberration in eyes with IOLs, Barbero et al $^{12}$ showed that spherical aberration increased as a function of the IOL power. The present study also demonstrates a similar relationship and extends this relationship by providing a mathematical relationship between the postoperative spherical aberration and the power of the IOL.

The aims of the study were 1) to characterize and measure the ocular aberrations in eyes implanted with IOLs, and 2) to study any correlation between postoperative aberrations and surgical factors.

\section{PATIENTS AND METHODS}

This study analyzed 62 eyes from patients diagnosed with cataract aged between 40 and 74 years who underwent phacoemulsification with implantation of a foldable hydrophobic acrylic IOL (Acrysof MA60BM; Alcon Laboratories Inc, Ft Worth, Tex) through a selfsealing 4.1-mm frown incision between the 10 and 11 o'clock meridians. The power of the IOLs ranged from 10.5 to 26.0 diopters. Eyes with any known ocular pathology (other than cataract) or previous ocular surgery were excluded from the study. Eyes that suffered any complication during surgery, including the loss of an intact capsulorrhexis or a tear in the posterior capsule, were also excluded. The study was approved by the in- stitutional review board and an informed consent was obtained from all patients.

The ALLEGRETTO WAVE Analyzer (WaveLight Laser Technologies AG, Erlangen, Germany) was used to measure each patient's wave aberration. Measurements were taken 2 weeks after surgery, when the eyes were believed to have attained refractive stability. A laser diode (wavelength of $532 \mathrm{~nm}$ ) was used to produce a collimated bundle of light, which was then split into a group of parallel rays by means of a mask with a regular matrix of small apertures. The retinal image of the spot pattern was photographed by a charged couple device camera.

The coordinates of the geometric centers of all imaged retinal spots were determined by image processing software and computed by numerical fitting to a Zernike expansion. The Zernike coefficients provided by the aberrometer were converted to the notation recommended by the Optical Society of America (OSA) standard committee, ${ }^{20}$ using the normalization factors provided by the manufacturers. The 62 eyes analyzed in this study included 31 right eyes and 31 left eyes. The signs of all modes with odd symmetry about the y-axis were negated in the left eyes to allow all eyes to be analyzed together. Measurements were made with a pupil diameter of at least $6 \mathrm{~mm}$. Only well-centered images were chosen for analysis. Zernike coefficients through the 6th order were measured and the rootmean-square (RMS) wavefront error of each order was calculated. Higher order aberrations included the 3rd through 6th order aberrations.

These coefficients were compared with those of a normal dataset of 82 eyes of healthy young myopes aged 19 to 48 years (mean 26.6 \pm 4.8 years) with refractive errors ranging from -0.75 to $-8.50 \mathrm{D}$ who attended our refractive surgery clinic.

\section{RESULTS}

Figure 1 shows the mean values of all Zernike coefficients from 3rd to 6th order, across a 6-mm pupil with error bars for the postoperative pseudophakic eyes. The 1st and 2nd order aberrations have been deleted. The most predominant aberrations that were statistically significantly greater than zero were spherical aberration $\left(Z_{4}^{0}\right)$ measuring $0.37 \pm 0.16 \mu \mathrm{m}(P \leqslant .001)$, trefoil $\left(Z_{3}^{-3}\right)$ measuring $-0.11 \pm 0.23 \mu \mathrm{m}(P=.001)$, and vertical coma $\left(Z_{3}^{-1}\right)$ measuring $-0.13 \pm 0.22 \mu \mathrm{m}(P<.001)$.

Figure 2 shows the corresponding RMS values of each order and the overall higher order RMS error in this group of pseudophakic eyes. The RMS of 3rd order measured $0.44 \pm 0.25 \mu \mathrm{m}$ and 4 th order measured $0.45 \pm 0.23 \mu \mathrm{m}$. The RMS of total higher order aberrations was $0.67 \pm 0.34 \mu \mathrm{m}$. 


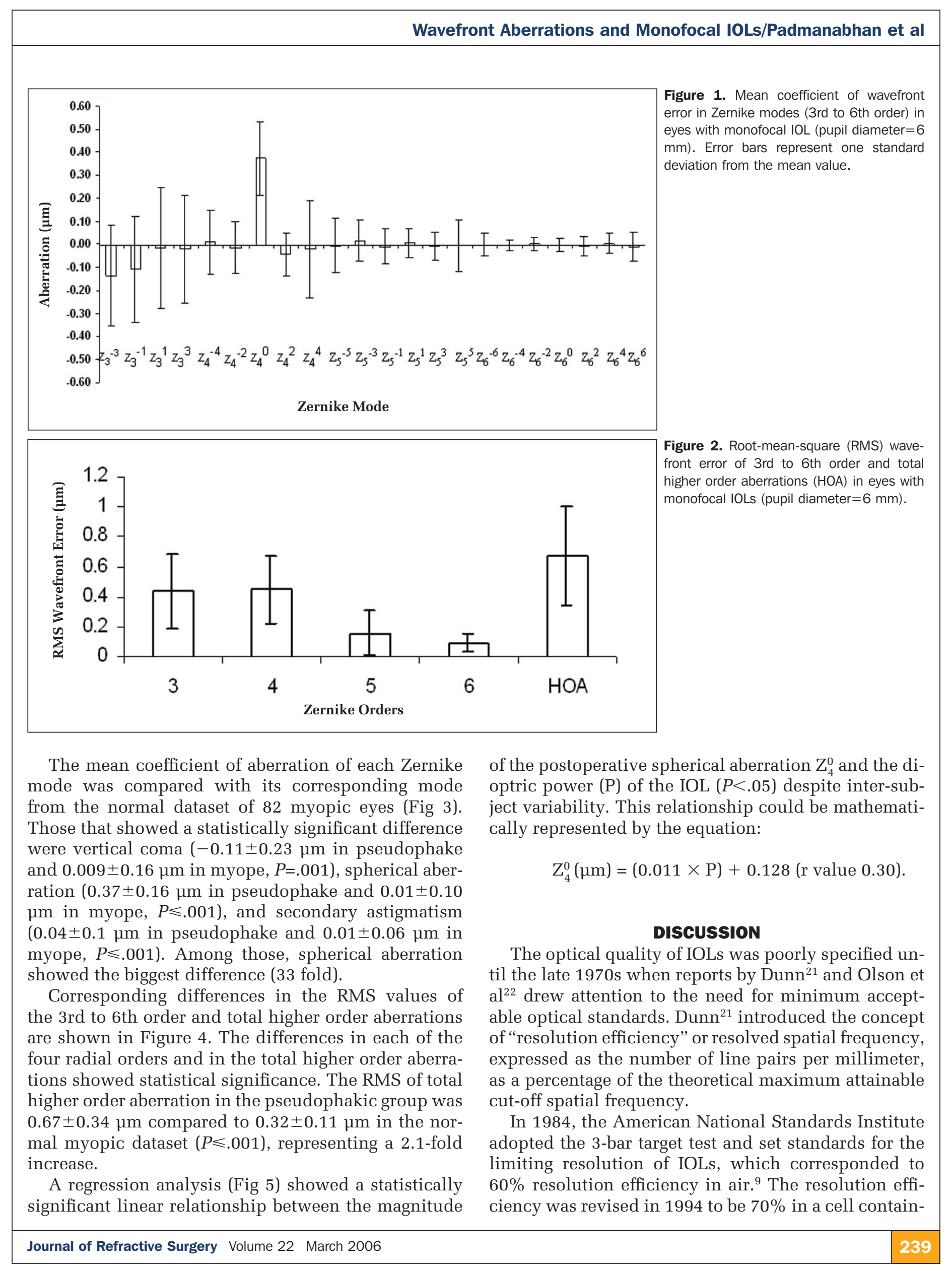




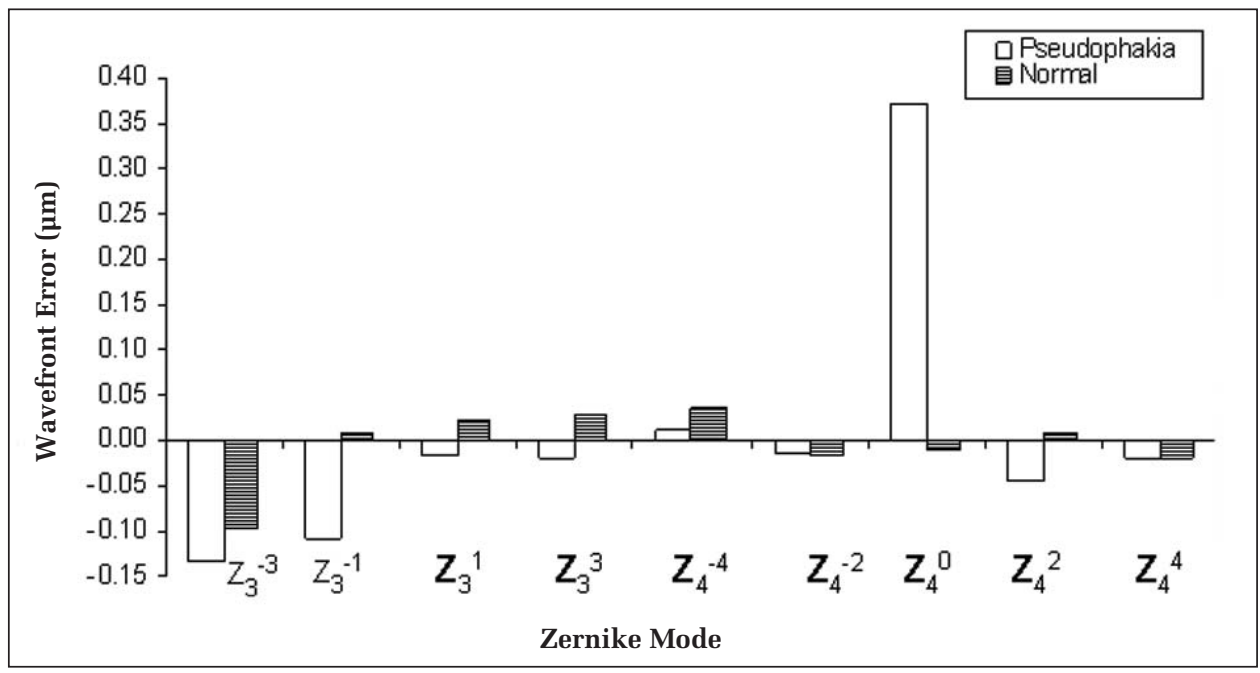

Figure 3. Comparison of mean coefficient of wavefront error of individual Zernike modes $\left(Z_{3}^{3}\right.$ to $\left.Z_{4}^{4}\right)$ in pseudophakic eyes with normal myopic dataset (pupil diameter $=6 \mathrm{~mm}$ ). Age of pseudophakes $=40$ to 74 years and age of normal myopes $=19$ to 48 years

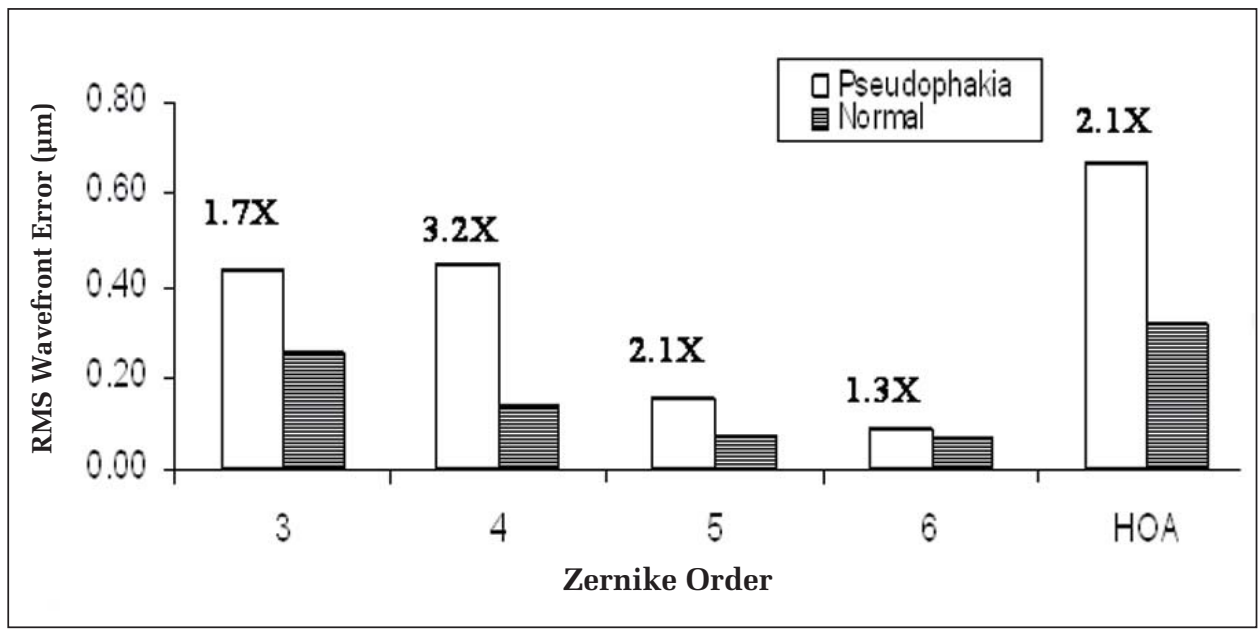

Figure 4. Comparison of RMS wavefront error of 3rd to 6th order and total higher order aberrations (HOA) between pseudophakic eyes and normal myopic eyes (pupil diameter $=6 \mathrm{~mm}$ ). Numerals indicate how many times more the magnitude of one is compared to the other.

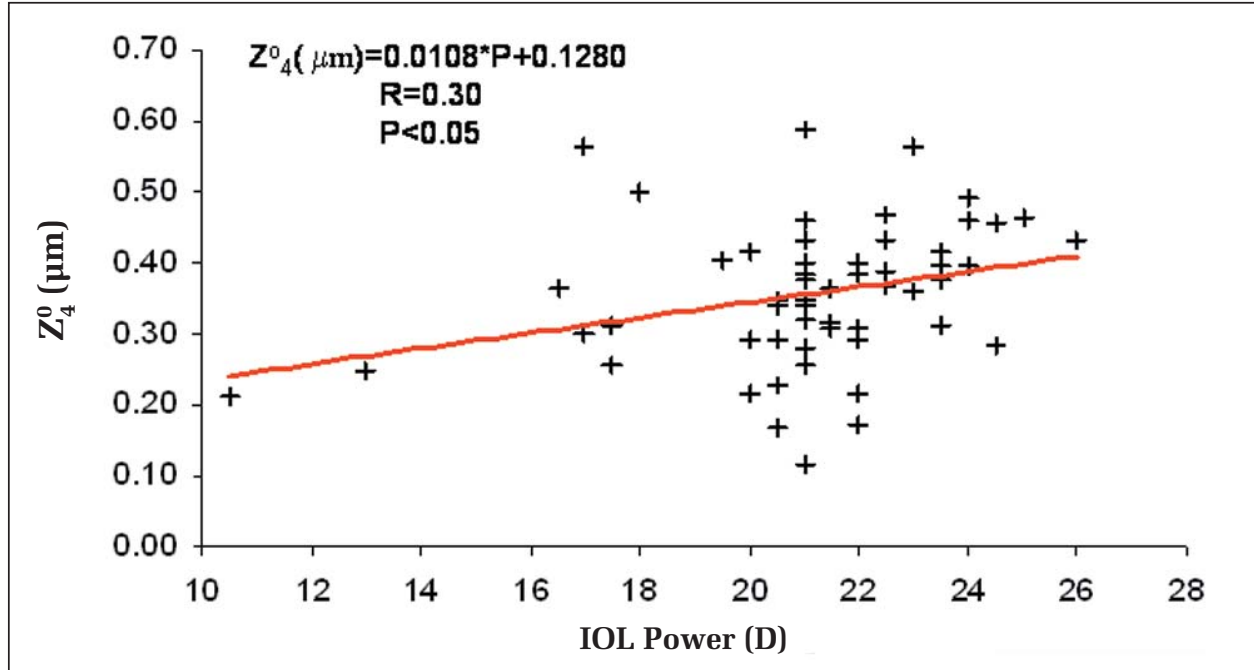

Figure 5. Regression analysis illustrating the relationship between the postoperatively measured value of spherical aberration $\left(Z_{4}^{0}\right)$ and the power of the IOL $(P)$ in pseudophakic eyes. The equation is: $Z_{4}^{0}=(0.011 \times P)+0.128$.

ing a liquid with a refractive index of $1.336 .{ }^{10}$ With modern methods of measuring image quality, MTF curves could be generated as well. Inter-laboratory testing has shown that MTF measurements using a model eye have better repeatability and reproducibility than the 3-bar target test. ${ }^{9}$ The commonly applied criterion of $60 \%$ resolution in air corresponds to $0.43 \mathrm{MTF}$ units at 100 lines $/ \mathrm{mm}$ in a model eye. All IOLs marketed today are expected to meet these criteria but unfortunately do not account for the effects of spherical aber- 


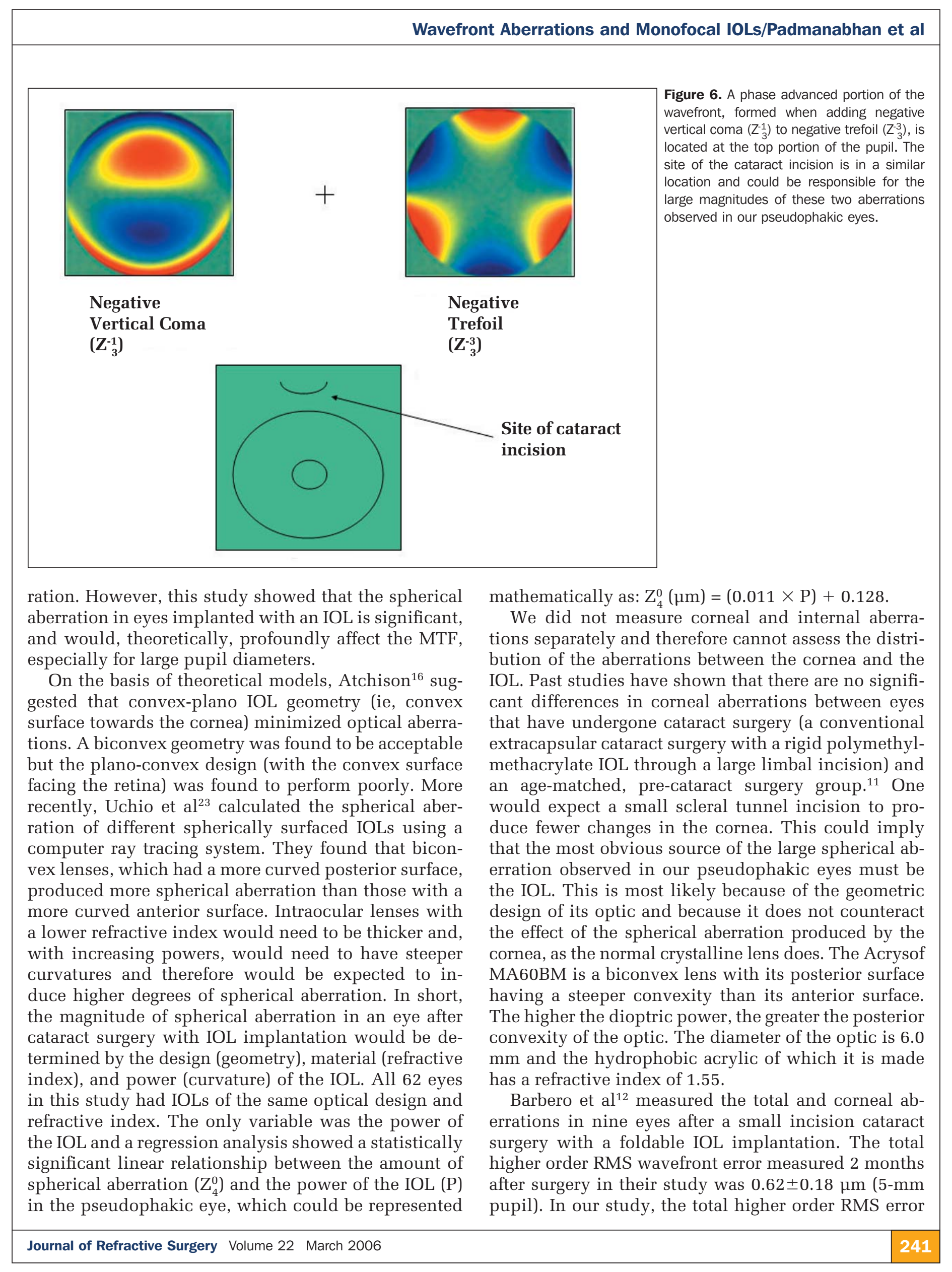


2 weeks after IOL implantation was $0.67 \pm 0.33 \mu \mathrm{m}$ (6-mm pupil), which correlates well with the results of Barbero et al.

Higher order aberrations in our dataset of pseudophakic eyes were 2.1 times larger than in young myopic eyes. It is well known that optical aberrations increase with age ${ }^{24}$ and comparisons of aberrations following cataract surgery with aberrations in a younger population of normal eyes could be misleading. Although it would have been ideal to compare postoperative aberrations to preoperative values within the same patients, there is considerable difficulty in obtaining wavefront measurements in eyes with a cataractous lens due to scatter and lens opacities. We did not have a dataset of normal healthy eyes in the same age group as that of the pseudophakic eyes, and therefore could not make such an age-matched comparison among our own population.

However, if racial differences are ignored, the results of other studies on patients of similar age groups as our own could be extrapolated to provide an estimate for the types of aberrations indicative of normal, healthy eyes in the same age range. Artal et $\mathrm{al}^{24}$ recorded an average higher order RMS wavefront error of $0.7 \mu \mathrm{m}$ for a 6-mm pupil. The higher order aberrations in our pseudophakic eyes for a 6-mm pupil (mean RMS of $0.67 \pm 0.34 \mu \mathrm{m}$ ) were not statistically significantly different from the higher order RMS values for the normal, similarly aged, healthy eyes reported by Artal et al. This observation is in agreement with similar conclusions by Barbero et al. ${ }^{12}$

This study showed a significant negative mean value for vertical coma $\left(\mathrm{Z}_{3}^{-1}=-0.11 \pm 0.23 \mu \mathrm{m}\right)$ and trefoil $\left(Z_{3}^{-3}=-0.13 \pm 0.22 \mu \mathrm{m}\right)$ in the pseudophakic eyes 2 weeks after cataract surgery. Combining these two aberrations into a single wavefront profile, one would notice the phase-advanced portion of the wavefront to correspond to the site of the cataract incision wound (Fig 6). Further studies on the effect of varying sites and sizes of incisions on aberrations may be required to improve our understanding of such possible associations.

\section{REFERENCES}

1. Leaming DV. Practice styles and preferences of ASCRS members-1999 survey. J Cataract Refract Surg. 2000;26:913-921.

2. Watson A, Sunderraj P. Comparison of small-incision phacoemulsification with standard extracapsular cataract surgery: post-operative astigmatism and visual recovery. Eye. 1992;6:626-629.

3. Porter J, Guirao A, Cox IG, Williams DR. Monochromatic aberrations of the human eye in a large population. J Opt Soc Am A. 2001;18:1793-1803.

4. Howland HC, Howland B. A subjective method for the mea- surement of monochromatic aberrations of the eye. J Opt Soc Am. 1997;67:1508-1518.

5. Applegate RA, Marsack JD, Ramos R, Sarver EJ. Interaction between aberrations to improve or reduce visual performance. J Cataract Refract Surg. 2003;29:1487-1495.

6. Thibos LN, Hong X. Clinical applications of the Shack-Hartmann aberrometer. Optom Vis Sci. 1999;76:817-825.

7. Simpson MJ. Optical quality of intraocular lens. J Cataract Refract Surg. 1992;18:86-94.

8. Norrby NE. Standardized methods for assessing the imaging quality of intraocular lenses. Appl Opt. 1995;34:7327-7333.

9. Norrby NE, Grossman LW, Geraghty ED, Kreiner CF, Mihori M, Patel AS, Portney V, Silberman DM. Determining the imaging quality of intraocular lens. J Cataract Refract Surg. 1998;24:703-714.

10. American National Standard for Ophthalmics-Intraocular Lenses-Optical and Physical Requirements, ANSI Z 80.7-1994. New York, NY: American National Standards Institute; 1994.

11. Guirao A, Redondo M, Geraghty E, Piers P, Norrby S, Artal P. Corneal optical aberrations and retinal image quality in patients in whom monofocal intraocular lenses were implanted. Arch Ophthalmol. 2002;120:1143-1151.

12. Barbero S, Marcos S, Jimenez-Alfaro I. Optical aberrations of intraocular lenses measured in vivo and in vitro. J Opt Soc Am A. 2003;20:1841-1851.

13. Navarro R, Ferro M, Artal P, Miranda I. Modulation transfer function of eyes implanted with intraocular lenses. Appl Opt. 1993;32:6359-6367

14. Artal P, Guirao A. Contributions of the cornea and the lens to the aberrations of the human eye. Opt Lett. 1998;23:1713-1715.

15. Artal P, Guirao A, Berrio E, Williams DR. Compensation of corneal aberrations by the internal optics in the human eye. J Vis. 2001;1:1-8.

16. Atchison DA. Optical design of intraocular lenses, I: on-axis performance. Optom Vis Sci. 1989;66:492-506.

17. Olsen T, Corydon L. Contrast sensitivity in patients with a new type of multifocal intraocular lens. J Cataract Refract Surg. 1990;16:42-46.

18. Artal P, Marcos S, Navarro R, Miranda I, Ferro M. Through focus image quality of eyes implanted with monofocal and multifocal intraocular lenses. Opt Eng. 1995;34:772-779.

19. Miller JM, Anwaruddin A, Straub J, Schwiegerling J. Higher order aberrations in normal, dilated, intraocular lens, and laser in situ keratomileusis corneas. J Refract Surg. 2002;18:S579-S583.

20. Thibos LN, Applegate RA, Schwiegerling JT, Webb R, VST Members. Standards for reporting the optical aberrations of eyes. In: Lakshminarayanan V, ed. Vision Science and Its Applications. Vol. 35 of OSA Trends in Optics and Photonics series. Washington, DC: Optical Society of America; 2000:110-130.

21. Dunn MJ. The resolving power of intraocular lens implants. J Am Intraocul Implant Soc. 1978;4:126-129.

22. Olson RJ, Kolodner H, Kaufman HE. The optical quality of currently manufactured intraocular lenses. Am J Ophthalmol. 1979;88:548-555.

23. Uchio E, Ohno S, Kusakawa T. Spherical aberrations and glare disability with intraocular lenses of different optical design. J Cataract Refract Surg. 1995;21:690-696.

24. Artal P, Berrio E, Guirao A, Piers P. Contribution of the cornea and internal surfaces to the change of ocular aberrations with age. J Opt Soc Am A. 2002;19:137-143. 